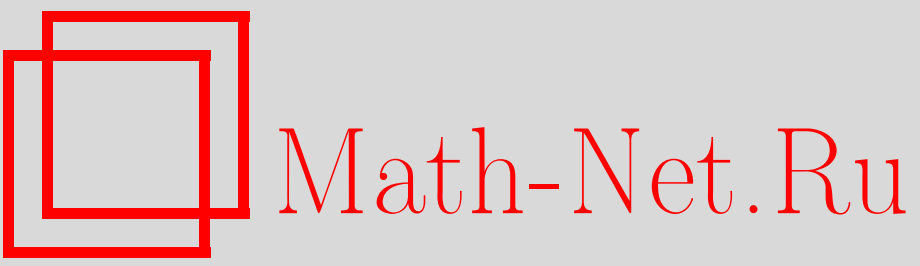

В. А. Зорич, Устранимая особенность квазиконформного погружения, УМН, 2001, том 56, выпуск 4, 147-148

DOI: https://doi.org/10.4213/rm426

Использование Общероссийского математического портала Math-Net.Ru подразумевает, что вы прочитали и согласны с пользовательским соглашением

http://www.mathnet.ru/rus/agreement

Параметры загрузки:

IP : 54.198 .64 .247

26 апреля 2023 г., 08:47:01 


\title{
УСТРАНИМАЯ ОСОБЕННОСТЬ КВАЗИКОНФОРМНОГО ПОГРУЖЕНИЯ
}

\author{
В. А. Зорич
}

Мы показываем, что в размерностях $n>2$ изолированная особенность квазиконформного погружения риманова многообразия в односвязное риманово многообразие той же размерности всегда устранима.

Теорема. Пусть $M^{n}$ и $N^{n}$ - римановы многообразия размерности $n$, о - точка на $M^{n}$ и $\dot{M}^{n}:=M^{n} \backslash\left\{\right.$ o\}. Пусть $f: \dot{M}^{n} \rightarrow N^{n}$ - квазиконформное погружение. Если многообразие $N^{n}$ односвязно, $a n \geqslant 3$, то отображение $f$ ингективно в некоторой проколотой окрестности точки о; при этом особенность либо устраняется непосредственно, либо әтого можно добиться, пополняя $N^{n}$ единственной точкой.

Говоря о погружении или иммерсии, мы будем иметь в виду локально инъективное (локально обратимое) отображение. Квазиконформность погружения подразумевает ограниченность его коэффициента квазиконформности. Устранение особенности предполагается в категории квазиконформных погружений.

Доказательство теоремы получается цепочкой утверждений.

УтвеРЖДЕНИЕ 1. Если иммерсия $f: \dot{M}^{n} \rightarrow N^{n}$ не имеет в особой точке ни конечного асимптотического значения, ни бесконечного предела, то существует идущий в особую точку периодический путь.

Здесь $f$ - произвольная, не обязательно квазиконформная иммерсия, а $M^{n}$ и $N^{n}$ - произвольные, не обязательно римановы многообразия размерности $n \geqslant 1$, которые, без ограничения общности, можно считать связными. Бесконечность предела означает, что по мере приближения точки $x \in \dot{M}^{n}$ к особой точке $o \in M^{n}$ ее образ $f(x)$ окончательно покидает любую компактную часть многообразия $N^{n}$. Наконец, периодичность пути означает, что он последовательно накрывает один и тот же цикл на $N^{n}$. Асимптотическое значение - это предел вдоль некоторого пути, идущего в особую точку.

Теперь доказательство. При указанных условиях для каждой проколотой окрестности $\dot{V}$ особой точки имеет место равенство $f(\dot{V})=N^{n}$ (см. предложение 1 в [1]). Это уже дает возможность построить идущий в особую точку периодический путь.

УтвеРЖДЕнИЕ 2. Если многообразие $N^{n}$ односвязно, то иммерсия $f: \dot{M}^{n} \rightarrow N^{n}$ имеет в особой точке либо конечное асимптотическое значение, либо бесконечный предел.

Заметим, что ни наличие конечного предела, ни конечная кратность или, тем более, инъективность $f$ в окрестности особой точки здесь не утверждаются (см., например, $z \mapsto \exp (1 / z))$.

Проведя некоторые построения, мы покажем, что если $N^{n}$ односвязно, то стягивание в точку цикла, накрьваемого периодическим путем из утверждения 2 , приводит к утверждению 3 .

Пусть $U$ - гомеоморфная стандартному $n$-мерному замкнутому диску (шару) окрестность точки $о$ в $M^{n} ; \partial U$ - граница $U$ (топологическая $(n-1)$-мерная сфера), которую мы будем также обозначать через $S^{n-1} ;$ и пусть $\dot{U}:=U \backslash\{o\}$.

Предположим, что иммерсия $f: \dot{U} \rightarrow N^{n}$ не имеет в особой точке ни конечного асимптотического значения, ни бесконечного предела. Тогда по утверждению 2 существует идущий в особую точку периодический путь, накрьвающий некоторый одномерньй цикл $\gamma$ в $N^{n}$.

Допустим, что нам удалось подклеить к $\dot{U}$ вдоль $S^{n-1}=\partial U n$-мерный диск и продолжить $f$ до иммерсии $F: \dot{S}^{n} \rightarrow N^{n}$ проколотой в точке $o$ сфферы $S^{n}$. Тогда препятствием к накрытию гомотопии цикла $\gamma$ в точку может быть только выход прообраза на точку $o \in S^{n}$. Но в этом случае

Работа выполнена при поддержке Российского фонда фундаментальных исследований (грант № 99-01-00969). 
у $f$ появляется конечное асимптотическое значение. Если же накрытие гомотопии не имело препятствий, то $f$ оказьвается однолистньм (инъективным) в проколотой окрестности точки $о$. Но если однолистное отображение $f$ не имеет конечного асимптотического значения, то, очевидно, у него есть бесконечньй предел в точке $o$.

Осталось разобраться с подклейкой диска и продолжением иммерсии.

Если иммерсия $f: \dot{U} \rightarrow N^{n}$ не имеет в особой точке ни конечного асимптотического значения, ни бесконечного предела, то, как уже отмечалось, для каждой проколотой окрестности $\dot{V} \subset U$ особой точки имеет место равенство $f(\dot{V})=N^{n}$. Возьмем точку $s \in\left(S^{n-1}=\partial U\right)$ и сходящуюся к $о$ последовательность точек $s_{k}, k=1,2, \ldots$, таких, что $f\left(s_{k}\right)=f(s)$. В каждой точке $s_{k}$, следуя параметризации $f \mid S^{n-1}$, построим свой экземпляр $S_{k}^{n-1}$ сферы $S^{n-1}$ над $f\left(S^{n-1}\right)$.

Сферы $S_{k}^{n-1}, k=1,2, \ldots$, не могут отделять $S^{n-1}$ от точки $о$ : иначе многообразие $N^{n}$ окажется неодносвязным. Значит, они стягиваемы в пределах $\dot{U}$. Любая такая сфера ограничивает стандартный топологический $n$-мерный диск в $\dot{U}$. Его построение и учет ориентации обосновьвают возможность использванной выше подклейки к $\dot{U}$ диска и продолжения иммерсии. Утверждение 2 доказано.

Риманова, конформная или квазиконформная структура многообразий, квазиконформность иммерсии и ограничение на размерность многообразий используются в следующем заключительном утверждении.

УтвеРЖДЕНИЕ 3. Если иммерсия $f: \dot{M}^{n} \rightarrow N^{n}$ в односвязное многообразие $N^{n}$ квазиконформна, то при $n \geqslant 3$ отображсение $f$ инбективно в некоторой проколотой окрестности особой точки.

Доказывая утверждение 2 , мы установили, что либо отображение $f$ имеет конечное асимптотическое значение в особой точке, либо $f$ инъективно в окрестности особой точки и имеет в ней бесконечный предел.

Но в силу предложения 3 из [1] наличие конечного асимптотического значения у квазиконформной иммерсии $f: \dot{M}^{n} \rightarrow N^{n}$ при $n \geqslant 3$ также влечет за собой инъективность $f$ в некоторой окрестности особой точки (и устранимость такой особенности). Утверждение 3 доказано.

Теперь, когда инъективность отображения $f$ в окрестности особой точки установлена, сформулированная выше теорема вытекает из хорошо известных фактов (верных и при $n \geqslant 2$ ), относящихся к устранению изолированной особенности однолистного квазиконформного отображения.

В связи с обсуждавшимися здесь вопросами см. обзор [2] и цитированную там литературу, а также гл. 6 в книге [3].

\section{СПИСОК ЛИТЕРАТУРЫ}

[1] В. А. Зорич // Функц. анализ и его прил. 2000. Т. 34. №3. С. 37-48. [2] V. A. Zorich // Lecture Notes in Math. 1992. V. 1508. P. 131-148. [3] M. Gromov (with appendices by M. Katz, P. Pansu, and S. Semmes). Metric Structures for Riemannian and Non-Riemannian Spaces. Boston: Birkhäuser, 1999. 